\title{
The ARGO-YBJ detector and high energy GRBs
}

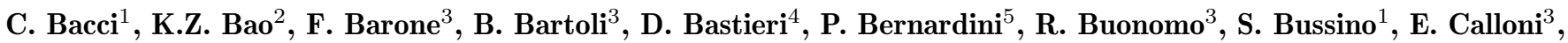
B.Y. Cao ${ }^{6}$, R. Cardarelli ${ }^{7}$, S. Catalanotti ${ }^{3}$, A. Cavaliere ${ }^{7}$, F. Cesaroni ${ }^{5}$, P. Creti $^{5}$, Danzengluobu ${ }^{8}$, B. D'Ettorre Piazzoli $^{3}$, M. De Vincenzi ${ }^{1}$, T. Di Girolamo ${ }^{3}$, G. Di Sciascio ${ }^{3}$, Z.Y. Feng ${ }^{9}$, Y. Fu ${ }^{6}$, X.Y. Gao ${ }^{10}$, Q.X. Geng ${ }^{10}$, H.W. Guo ${ }^{8}$, H.H. He ${ }^{11}$, M. He ${ }^{6}$, Q. Huang ${ }^{9}$, M. Iacovacci $^{3}$, N. Iucci ${ }^{1}$, H.Y. Jai ${ }^{9}$, F.M. Kong ${ }^{6}$, H.H. Kuang $^{11}$, Labaciren $^{8}$, B. Li $^{2}$, J.Y. Li ${ }^{6}$, Z.Q. Liu ${ }^{10}$, H. Lu ${ }^{11}$, X.H. Ma ${ }^{11}$, G. Mancarella ${ }^{5}$, S.M. Mari ${ }^{12}$, G. Marsella ${ }^{5}$, D. Martello ${ }^{5}$, D.M. Mei ${ }^{8}$, X.R. Meng ${ }^{8}$, L. Milano ${ }^{3}$, A. Morselli ${ }^{7}$, J. Mu$^{10}$, M. Oliviero ${ }^{13}$, P. Padovani $^{7}$, M. Panareo $^{5}$, M. Parisi ${ }^{1}$, G. Pellizzoni ${ }^{1}$, Z.R. Peng ${ }^{11}$, P. Pistilli ${ }^{1}$, R. Santonico ${ }^{7}$, G. Sartori ${ }^{4}$, C. Sbarra ${ }^{4}$, G. Severino ${ }^{13}$, P.R. Shen ${ }^{11}$, R. Sparvoli ${ }^{7}$, C. Stanescu ${ }^{1}$, J. Su ${ }^{11}$, L.R. Sun ${ }^{2}$, S.C. Sun ${ }^{2}$, A. Surdo ${ }^{5}$, Y.H. Tan ${ }^{11}$, S. Vernetto ${ }^{14}$, M. Vietri ${ }^{1}$, C.R. Wang ${ }^{6}$, H. Wang ${ }^{11}$, H.Y. Wang ${ }^{11}$, Y.N. Wei ${ }^{2}$, H.T. Yang ${ }^{12}$, Q.K. Yao ${ }^{2}$, G.C. Yu ${ }^{9}$, X.D. Yue ${ }^{2}$, A.F. Yuan ${ }^{8}$, H.M. Zhang ${ }^{11}$, J.L. Zhang ${ }^{11}$, N.J. Zhang ${ }^{6}$, T.J. Zhang ${ }^{10}$, X.Y. Zhang ${ }^{6}$, Zhaxisangzhu ${ }^{8}$, Zhaxiciren ${ }^{8}$, and Q.Q. $\mathbf{Z h u}^{11}$

1 INFN and Dipartimento di Fisica dell'Università di Roma Tre, Italy

2 Zhenghou University, Henan, China

3 INFN and Dipartimento di Fisica dell'Università di Napoli, Italy

${ }^{4}$ INFN and Dipartimento di Fisica dell'Università di Padova, Italy

5 INFN and Dipartimento di Fisica dell'Università di Lecce, Italy

${ }^{6}$ Shangdong University, Jinan, China

7 INFN and Dipartimento di Fisica dell'Università di Roma "Tor Vergata", Italy

8 Tibet University, Lhasa, China

9 South West Jiaotong University, Chengdu, China

10 Yunnan University, Kunming, China

11 IHEP, Beijing, China

12 Universitá della Basilicata, Potenza, Italy

13 Osservatorio Astronomico di Capodimonte, Napoli, Italy

14 Istituto di Cosmogeofisica del CNR and INFN, Torino, Italy e-mail: vernetto@lngs.infn.it

Received December 29, 1998; accepted April 21, 1999

\begin{abstract}
ARGO-YBJ (Astrophysical Radiation with Ground-based Observatory at YangBaJing) is a detector optimized to study small size air showers. It consists of a layer of Resistive Plate Counters (RPCs) covering an area of $\sim 6500 \mathrm{~m}^{2}$ and will be located in the Yangbajing Laboratory (Tibet, China) at $4300 \mathrm{~m}$ a.s.l. ARGO-YBJ will be devoted to a wide range of fundamental issues in cosmic rays and astroparticle physics, including in particular gamma-ray astronomy and gamma-ray bursts physics in the range $10 \mathrm{GeV} \div 500 \mathrm{TeV}$. The sensitivity of ARGO-YBJ to detect high energy GRBs is presented.
\end{abstract}

Key words: gamma-ray: bursts

\section{Introduction}

The study of the GeV - TeV component of gamma-ray bursts is of great importance to understand the accelera-

Send offprint requests to: S. Vernetto tion mechanisms and the sources physical conditions. The detection of GeV gamma-rays by EGRET during some intense GRBs (Catelli et al. 1997) suggests the possibility that a high energy component could be present in all events. Furthermore several models predict $\mathrm{GeV}$ and $\mathrm{TeV}$ emission, sometimes correlated with UHECRs production (see Baring 1997, for a review). Due to the low fluxes and the small sensitive areas of satellite experiments, gammarays of energy larger than a few tens of $\mathrm{GeV}$, must be detected by ground based experiments located at mountain altitude measuring the secondary particles generated by gamma-rays in the atmosphere. At energies $E<10 \mathrm{TeV}$ the number of particles reaching the ground is to small to reconstruct the shower parameters using a standard air shower array, made of several detectors spread over large areas. On the contrary, a detector consisting of a full coverage layer of counters, providing a high granularity sampling of all particle showers, can successfully measure arrival direction and primary energy of small showers, 
allowing the study of the unexplored range of gamma energies between $20 \mathrm{GeV}$ and $300 \mathrm{GeV}$ (Abbrescia et al. 1996).

\section{The ARGO-YBJ detector}

ARGO-YBJ is an air shower detector optimized to observe small size showers, to be constructed in the Yangbajing Laboratory (Tibet, China) at an altitude of $4300 \mathrm{~m}$ a.s.l. The experiment consists of a $\sim 71 \times 74 \mathrm{~m}^{2}$ core detector realised with a single layer of RPC's $(\sim 90 \%$ of active area), surrounded by an outer detector $(\sim 30 \%$ of active area) for a total size of $\sim 100 \times 100 \mathrm{~m}^{2}$. A lead converter $0.5 \mathrm{~cm}$ thick will cover uniformly the RPC plane in order to increase the number of charged particles by conversion of shower photons in $\mathrm{e}^{ \pm}$and to reduce the time spread of the shower front (Bacci et al. 1998). ARGOYBJ can image with high efficiency and sensitivity atmospheric showers initiated by primaries of energies in the range $10 \mathrm{GeV} \div 500 \mathrm{TeV}$. Its main physics goals are (Abbrescia et al. 1996): Gamma-astronomy at $\sim 100 \mathrm{GeV}$ energy threshold, with a sensitivity to detect unidentified point sources of intensity as low as $10 \%$ of the Crab Nebula; Gamma-Ray Burst physics, extending the satellite measurements at energies $E>10 \mathrm{GeV} ; \bar{p} / p$ ratio in the $\mathrm{TeV}$ energy range; Sun and heliosphere physics. The detector assembling should start in 2000 and data taking with the first $\sim 750 \mathrm{~m}^{2}$ of RPC's in 2001 .

\section{Sensitivity to high energy GRBs}

A high energy GRB is detectable if the number of air showers from the gamma-rays is significant larger than the fluctuations of the background, due to showers from cosmic rays with arrival directions compatible with the burst position. A good angular resolution is of major importance in order to reduce the background and increase the detection sensitivity. The angular resolution and the effective area of ARGO-YBJ to detect gamma-rays as a function of the energy have been obtained by means of simulations. For gamma-rays with energy as low as $E \sim 10-20 \mathrm{GeV}$, the opening angle around the source direction in which $70 \%$ of the signal showers are contained is $\sim 5^{\circ}$.

To evaluate the sensitivity of ARGO-YBJ to detect GRBs, we considered a burst with a power law energy spectrum $\mathrm{d} N / \mathrm{d} E \propto E^{-\alpha}$ extending in the range $1 \mathrm{GeV} \div E_{\max }$, a duration $\Delta t=1 \mathrm{~s}$, and a zenith angle $\theta=20^{\circ}$. The burst will give a signal with a significance larger than 4 standard deviations if the energy fluence in the range $1 \mathrm{GeV} \div E_{\max }$ is larger than a minimum value $F_{\min }$. Figure 1 shows $F_{\min }$ as a function of $E_{\max }$ for 3 spectral slopes. For a generic duration $\Delta t$ the minimum fluences detectable are given by $F_{\min } \sqrt{\Delta t}$.

In the energy range considered the sensitivity is strongly dependent on the maximum energy of the spectrum $E_{\max }$. ARGO-YBJ can observe GRBs with energy fluences of a few $10^{-6} \mathrm{erg} \mathrm{cm}^{-2}$ if the energy spectrum extends at least up to $\sim 200 \mathrm{GeV}$ with a slope

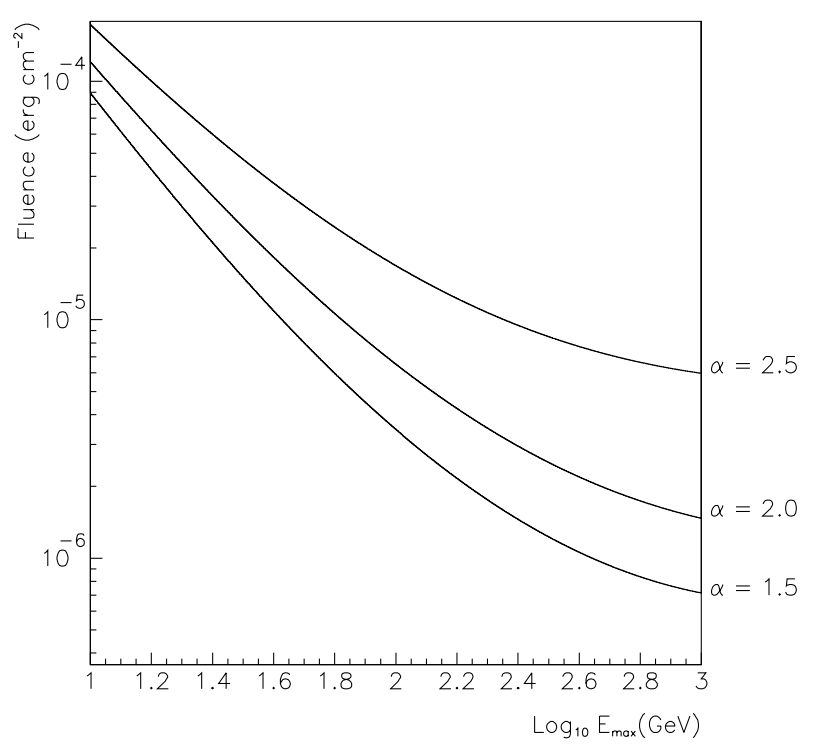

Fig. 1. The minimum energy fluence in the range $1 \mathrm{GeV} \div E_{\max }$ of a GRB detectable by ARGO-YBJ as a function of the maximum energy of the spectrum $E_{\max }$ for 3 spectral slopes

$\alpha \leq 2$; the minimum detectable fluence is $\sim 10^{-5}$ if $E_{\max } \sim 30 \mathrm{GeV}$.

This is of particular importance, since if GRB sources are located at cosmological distances, the high energy tail of the spectrum is affected by the $\gamma \gamma \rightarrow \mathrm{e}^{+} \mathrm{e}^{-}$interaction of gamma-rays with low energy starlight photons in the intergalactic space. According to Salomon \& Stecker (1998), at a distance corresponding to a redshift $z=0.1$ the absorption is almost negligible, while at $z=0.5(1.0)$ the absorption becomes important for photons of energy $E>100$ (50) GeV. These values give an idea of the possible maximum energy of the GRBs spectra as a function of their distance, and from Fig. 1 one can infer the maximum sensitivity of ARGO-YBJ to detect cosmological GRBs. The minimum observable fluences can be compared with the fluences measured by EGRET in the $1 \mathrm{MeV}-1 \mathrm{GeV}$ energy range: $F \sim 10^{-5} \div 10^{-4} \mathrm{erg} \mathrm{cm}^{-2}$ (Catelli et al. 1997). Since EGRET spectral slopes $\alpha$ are mostly $\sim 2$, one could expect fluences of the same order of magnitude at energies above $1 \mathrm{GeV}$. From Fig. 1 one can conclude that ARGO-YBJ could detect GRBs with the same intensity of those observed by EGRET provided that the energy spectrum extends up to few tens of $\mathrm{GeVs}$; the sensitivity increases by a factor $\sim 10$ for spectra extending up to $E_{\max } \sim 200 \mathrm{GeV}$.

\section{References}

Abbrescia, et al., 1996, Proposal of the ARGO experiment, http://www1.na.infn.it/wsubnucl/cosm/argo/argo.html

Bacci C., et al., 1998, Addendum to the ARGO Proposal, http://www1.na.infn.it/wsubnucl/cosm/argo/argo.html

Baring M.W., astro-ph9711256, 1997

Catelli J.R., et al., 1997, AIP Conf. Proc. 428, 309

Salomon M.H., Stecker F.W., 1998, ApJ 493, 547 